\title{
Factors related to abdominal pain in gastroparesis: contrast to patients with predominant nausea and vomiting
}

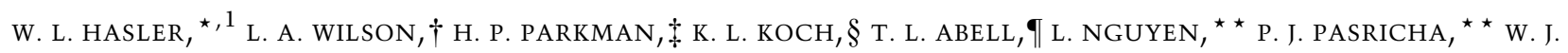

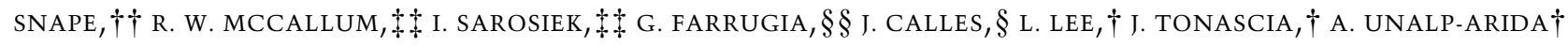
\& F. HAMILTON $\|$

${ }^{\star}$ University of Michigan, Ann Arbor, Michigan, USA

$\dagger$ †ohns Hopkins University, Baltimore, Maryland, USA

$\ddagger$ Temple University, Philadelphia, Pennsylvania, USA

$\S$ Wake Forest University, Winston-Salem, North Carolina, USA

qUniversity of Mississippi, Jackson, Mississippi, USA

**Stanford University, Palo Alto, California, USA

$\dagger \dagger$ California Pacific Medical Center, San Francisco, California, USA

\$†Texas Tech University Health Sciences Center, El Paso, Texas, USA

$\S \S$ Mayo Clinic, Rochester, Minnesota, USA

qqINational Institute of Diabetes and Digestive and Kidney Diseases, Bethesda, Maryland, USA

\begin{abstract}
Background Factors associated with abdominal pain in gastroparesis are incompletely evaluated and comparisons of pain vs other symptoms are limited. This study related pain to clinical factors in gastroparesis and contrasted pain/discomfort- with nausea/vomiting-predominant disease. Methods Clinical and scintigraphy data were compared in 393 patients from seven centers of the NIDDK Gastroparesis Clinical Research Consortium with moderate-severe (Patient Assessment of Upper Gastrointestinal Disorders Symptoms [PAGI-SYM] score $\geq 3$ ) vs none-mild (PAGI-SYM < 3) upper abdominal pain and predominant pain/discomfort vs nausea/vomiting. Key Results Upper abdominal pain was moderate-severe in $261(66 \%)$. Pain/discomfort was predominant in 81 (21\%); nausea/vomiting was predominant in 172 (44\%). Moderate-severe pain was more prevalent with
\end{abstract}

Address for Correspondence

William L. Hasler, MD, 3912 Taubman Center, 5362, Ann Arbor, MI 48109.

Tel: +734-936-4780; fax: +734-936-7392;

e-mail: whasler@umich.edu

Members of the gastroparesis clinical research consortium have been included in the appendix.

${ }^{1}$ All the authors belong to Writing Committee of The NIDDK Gastroparesis Clinical Research Consortium (GpCRC)

Received: 23 September 2012

Accepted for publication: 24 December 2012 idiopathic gastroparesis and with lack of infectious prodrome $(P \leq 0.05)$ and correlated with scores for nausea/vomiting, bloating, lower abdominal pain/ discomfort, bowel disturbances, and opiate and antiemetic use $(P<0.05)$, but not gastric emptying or diabetic neuropathy or control. Gastroparesis severity, quality of life, and depression and anxiety were worse with moderate-severe pain $(P \leq 0.008)$. Factors associated with moderate-severe pain were similar in diabetic and idiopathic gastroparesis. Compared to predominant nausea/vomiting, predominant pain/discomfort was associated with impaired quality of life, greater opiate, and less antiemetic use $(P<0.01)$, but similar severity and gastric retention. Conclusions $\Theta$ Inferences Moderate-severe abdominal pain is prevalent in gastroparesis, impairs quality of life, and is associated with idiopathic etiology, lack of infectious prodrome, and opiate use. Pain is predominant in one fifth of gastroparetics. Predominant pain has at least as great an impact on disease severity and quality of life as predominant nausea/vomiting.

Keywords diabetes mellitus, gastric emptying, gastroparesis, opiates, psychological dysfunction, quality of life.

\section{INTRODUCTION}

Clinical features of gastroparesis have been characterized by extensive investigations summarized in con- 
sensus publications. ${ }^{1,2}$ Nausea (prevalence $79 \%-93 \%$ ) and vomiting $(41 \%-68 \%)$ traditionally are considered the main symptoms of gastroparesis, but single-center studies report prevalences of abdominal pain ranging from $42 \%-89 \% .{ }^{2-8}$ In one series, pain was predominant in $44 \%$ while nausea and vomiting dominated in $51 \%{ }^{7}$ Sixty-one percent of physicians ranked postprandial pain as the most frequent symptom of gastroparesis in one report. ${ }^{9}$ The pathophysiology of pain in gastroparesis is poorly understood; available studies do not indicate a pathogenic role for delayed gastric emptying. ${ }^{3,10-14}$ Treatment trials have not focused on gastroparesis pain. Current practice centers on use of prokinetics, antidepressants, pain modulators, gastric stimulation, and opiates to manage gastroparesis-associated pain. ${ }^{7,8,15,16}$

The literature on gastroparesis pain exhibits deficiencies including small cohorts, use of non-validated surveys or scintigraphy methods, and limited assessments of pain effects on quality of life, psychological function, and medication use. ${ }^{3,6,7}$ Factors associated with pain have not been contrasted in diabetic and idiopathic disease. This is relevant as experts question if pain-predominant idiopathic gastroparesis should instead be considered to be functional dyspepsia. ${ }^{17}$ Among diabetics with gastroparesis, relations of pain to other diabetic complications or glycemic control are uncertain. Finally, clinical features in gastroparetics with predominant pain have not been contrasted to those with predominant nausea or vomiting, the more typical dominant symptoms of gastroparesis.

This study collected data using standardized instruments and diagnostic criteria to test hypotheses that abdominal pain is prevalent in gastroparesis and is the predominant symptom in a subset, that pain severity in gastroparesis is associated with other clinical parameters and impaired quality of life, but does not relate to degrees of gastric emptying impairment, and that pain predominance in gastroparesis has a distinct clinical profile compared to nausea or vomiting dominant disease. To test these hypotheses, we addressed these specific aims: (i) quantify the prevalence and severity of upper abdominal pain and discomfort in gastroparesis, (ii) relate pain/discomfort severity to a range of clinical factors, other measures of gastroparesis severity, quality of life, psychological function, gastric emptying, and medication use, (iii) ascertain if pain presentations depend on diabetic vs idiopathic etiology and delineate if pain in diabetic gastroparesis relates to diabetic neuropathy or glycemic control, and (iv) compare factors in patients reporting pain/discomfort vs nausea/vomiting as the predominant symptom to assess if different dominant symptoms have similar impact. These analyses, including relation of pain severity to other factors and characterization of a gastroparesis subset with pain predominance, were designed to provide a foundation for future investigations into the pathogenesis and management of abdominal pain in gastroparesis.

\section{MATERIALS AND METHODS}

\section{Patient population}

A total of 393 gastroparesis patients (age $>18$ years) were recruited at seven centers of the NIDDK Gastroparesis Clinical Research Consortium into a Gastroparesis Registry from January 2007 through March 2010 (ClinicalTrials.gov Identifier: NCT00398801) using consistent inclusion and exclusion criteria. All patients reported symptoms of gastroparesis of at least 12 weeks duration (not necessarily contiguous with varying degrees of nausea, vomiting, abdominal pain, early satiety, postprandial fullness) and had completed a 4-h scintigraphic low fat Egg Beaters gastric emptying study with evidence of delay $(>60 \%$ retention at $2 \mathrm{~h}$ and/or $>10 \%$ retention at $4 \mathrm{~h}$ ) within the 6 months prior to enrollment as well as upper gastrointestinal endoscopy to exclude organic diseases potentially causative of symptoms (e.g. ulcer disease, malignancy, gastric outlet obstruction) within the 12 months prior to enrollment. ${ }^{18}$ Patients were excluded if other conditions were present that could explain their symptoms (e.g. pyloric or intestinal obstruction, active inflammatory bowel disease, eosinophilic gastroenteritis, neurologic disease, active liver or renal disease, other metabolic causes, or prior fundoplication, gastric resection, or pyloroplasty). Institutional Review Board approval was obtained at all centers; patients provided written informed consent.

\section{Data acquisition}

Principal investigators, study coordinators, and patients completed standardized surveys on Registry enrollment. Demographic variables from Registration and Baseline Medical History forms included age, gender, self-reported ethnicity (Hispanic or Latino vs not Hispanic and not Latino) and race (American Indian or Alaska native vs Asian vs Black or African American vs. Native Hawaiian or other Pacific Islander vs. White vs. Patient Refused), gastroparesis etiology, symptom onset acuity, reported initial infectious prodromes, and prokinetic (metoclopramide, erythromycin, domperidone, clarithromycin, azithromycin, pyloric botulinum toxin), antiemetic (prochlorperazine, promethazine, trimethobenzamide, meclizine, ondansetron, granisetron, dolasetron, aprepitant), opiate, neuropathic pain modulator (gabapentin, pregabalin, topiramate), and antidepressant (amitriptyline, nortriptyline, desipramine, imipramine, buproprion, venlafaxine, duloxetine, fluoxetine, paroxetine, sertraline, citalopram, escitalopram, mirtazapine, trazodone) medication use. Additional information relating to the presence or absence of self-reported neuropathy (as recorded on the Baseline Medical History form) and levels of hemoglobin Alc was obtained in the diabetic patients. Gastric retentions at 2 and $4 \mathrm{~h}$ were measured.

Gastroparesis severity was assessed both by investigators and patients. Clinician-rated gastroparesis severity was assessed as grade 1, 2, or 3 using an expert-proposed stratification: grade 1 (easily controlled symptoms with maintenance of weight on a regular diet), grade 2 (moderate symptoms partly controlled by 
daily medications, but with maintenance of nutrition with dietary modification), and grade 3 (symptoms that are medication-refractory, if frequent physician and emergency department visits or hospitalizations are noted, and/or if oral nutrition is impossible). ${ }^{2}$ Patient-rated severity was quantified by modified Patient Assessment of Upper Gastrointestinal Disorders Symptoms (PAGI-SYM) questionnaires that enumerate 22 symptoms from 0 (no symptoms) to 5 (most severe). ${ }^{19}$ Upper and lower abdominal pain and discomfort were separately scored. Overall disease severity was determined by the Gastroparesis Cardinal Symptom Index (GCSI), which includes nine questions from the PAGI-SYM. ${ }^{20}$ GCSI subscale scores for nausea/vomiting (mean of scores for nausea [feeling sick to your stomach as if you were going to vomit or throw up], retching [heaving as if to vomit, but nothing comes up], and vomiting), postprandial fullness/early satiety (mean of scores for stomach fullness, not able to finish a normal-sized meal, feeling excessively full after meals, and loss of appetite), and bloating/distention (mean of scores for bloating [feeling like you need to loosen your clothes] and stomach or belly visibly larger) were calculated. The modified PAGI-SYM also contained bowel questions scoring constipation and diarrhea.

Measures of life quality and psychological function were quantified. Disease-specific quality of life was assessed by the Patient Assessment of Upper Gastrointestinal Disorders Quality of Life (PAGI-QOL) survey, which scores 30 factors from 0 (none of the time) to 5 (all of the time). ${ }^{21}$ Overall PAGI-QOL scores are means of all factors after reversing individual scores; an overall score of 0 represents poor quality of life. Subscores from the Short Form-36 (SF-36) were consolidated into separate component scores as follows: physical (physical functioning, role-physical, bodily pain, and general health) and mental (vitality, social functioning, role-emotional, and mental health). The Brief Pain Inventory (BPI) includes seven factors (general activity, mood, walking ability, normal work, relations with people, sleep, and enjoyment of life) quantifying interference produced by pain over the past day from 0 (does not interfere) to 10 (completely interferes). Overall pain interference scores were calculated from subscore means. Depression was quantified by the Beck Depression Inventory (BDI), which includes 21 questions pertaining to depression, cognition, and physical well-being. ${ }^{22}$ Anxiety was measured by the State and Trait Anxiety Inventory (STAI), which consists of 40 questions relating to state (temporary condition varying in intensity) and trait anxiety (general propensity to be anxious). ${ }^{23}$

\section{Pain severity and symptom predominance comparisons}

Initial comparisons stratified patients with moderate to severe upper abdominal pain or discomfort (score $\geq 3$ for either upper abdominal pain or discomfort) vs none to mild upper abdominal pain or discomfort (scores $<3$ for both upper abdominal pain and discomfort) severity. Separate analyses were performed for diabetic and idiopathic patients to determine if etiology influences pain impact. A total of 137 patients were deemed diabetic gastroparetics; 256 had idiopathic gastroparesis based on no diabetes or gastric surgery, normal hemoglobin Alc, and no other potential defined etiologies. Patients with other etiologies of gastroparesis were too few for analysis and were excluded. Clinical factors were compared in pain/discomfort severity subgroups.

For the second series of comparisons, subjects were stratified into abdominal pain/discomfort vs nausea/vomiting predominance and clinical factors were compared in the symptom predominance subgroups. Symptom predominance was written by patients in response to a query on the modified PAGI-SYM survey asking which symptom was the predominant one (this question is not part of the original PAGI-SYM). ${ }^{20}$ Pain/discomfort predominance was identified when one of the following was deemed predominant: upper abdominal pain (above the navel), upper abdominal discomfort (above the navel), lower abdominal pain (below the navel), lower abdominal discomfort (below the navel), abdominal pain location not specified, or abdominal discomfort location not specified. Nausea/vomiting predominance was identified when one of the following was deemed predominant: nausea [feeling sick to your stomach as if you were going to vomit or throw up], retching [heaving as if to vomit, but nothing comes up], or vomiting.

\section{Statistical analysis}

Factor differences were compared for those with upper pain/ discomfort scores of $\geq 3$ vs $<3$. Subanalyses were performed for diabetic and idiopathic gastroparetics. Additional analyses compared factors with predominant pain/discomfort vs. nausea/vomiting. Data presented are means $\pm \mathrm{SD}$, numbers $(\%)$, and differences in means or proportions. 95\% confidence intervals for differences in means were based on the t-distribution; intervals for differences in proportions used the Newcombe score method with continuity correction. ${ }^{24} P$ values were derived from chisquare tests for categorical variables, Fisher's exact tests for categorical variables with small expected numbers, or $t$-tests for continuous variables. Multiple logistic regression models for the outcome measures (upper pain/discomfort scores $\geq 3$ vs $<3$ and predominant abdominal pain/discomfort vs nausea/vomiting) included these covariates: age, gender, self-reported race and Hispanic ethnicity, etiology (idiopathic vs diabetic), onset acuity, initial infectious prodrome, two indicator variables representing clinician-rated severity (grades 2 and 3 vs grade 1), overall GCSI, PAGI-SYM constipation and diarrhea scores, PAGI-QOL, SF-36 physical component, BDI, STAI Y1, $2 \mathrm{~h}$ gastric retention, and medications (prokinetics, antiemetics, opiates, neuropathic pain modulators, antidepressants). SF-36 mental components were not included in models due to colinearity with PAGI-QOL; $2 \mathrm{~h}$ gastric retentions were included in models while $4 \mathrm{~h}$ measures were removed, and STAI Y1 measures were included, but Y2 values were removed. Hosmer-Lemeshow goodness-of-fit test indicated adequate fit for both models $(P=0.47$ and $P=0.84)$. Analyses were performed using SAS software (version 9.3, SAS Institute, Cary, NC) and Stata (Release 11.0, Stata Corporation, College Station, TX). ${ }^{25,26}$ Nominal, two-sided $P$ values were significant if $P<0.05$; no adjustments for multiple comparisons were made.

\section{RESULTS}

\section{Moderate-severe vs none-mild pain/discomfort}

Upper abdominal pain and discomfort ranged in severity in the whole group; $261(66 \%)$ and $277(70 \%)$ reported moderate or greater (PAGI-SYM score 3,4 , or 5) pain and discomfort, respectively; $288(73 \%)$ had either moderate or greater pain or discomfort (Fig. 1A).

Factors were compared with moderate to severe upper pain/discomfort vs none to mild severity (Table 1). PAGI-SYM upper pain and discomfort scores were $3.8 \pm 1.2$ and $4.0 \pm 0.9$, respectively, in the higher pain/discomfort group vs. $0.8 \pm 0.8$ and 

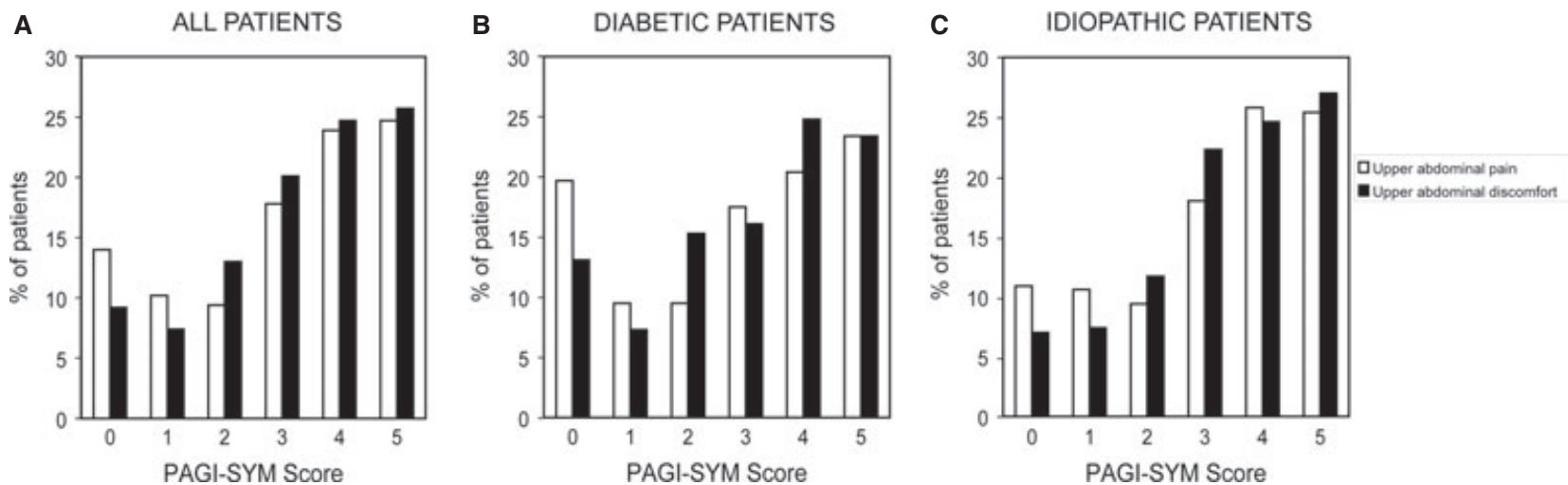

Figure 1 Percentages reporting upper abdominal pain (white bars) and discomfort (black bars) severity from 0 (none) to 5 (very severe) on the PAGI-SYM survey are plotted for all gastroparetics $(\mathrm{A})$ and for diabetic $(\mathrm{B})$ and idiopathic $(\mathrm{C})$ patients.

$1.1 \pm 0.9$ in the lower severity group (both $P<0.001$ ). The higher pain discomfort subgroup had a greater percentage with idiopathic etiology $(P=0.045)$ and approached significance to a higher percentage with female sex $(P=0.052)$. Pain/discomfort severity correlated with clinician-rated severity with a lower percentage with grade 1 gastroparesis reporting pain $\geq 3$ $(P=0.008)$. Overall GCSI scores; nausea/vomiting, fullness/early satiety, and bloating/distention subscale scores; and PAGI-SYM lower pain and discomfort, constipation, and diarrhea scores were greater with pain/discomfort scores $\geq 3$ (all $P \leq 0.001$ ). PAGI-QOL and SF-36 physical and mental component scores were lower with pain/discomfort scores $\geq 3$, while all BPI subscores; overall pain interference scores; and BDI depression and STAI Y1 state and Y2 trait anxiety scores were greater in the higher severity subgroup (all $P \leq 0.001$ ). Gastric retentions at 2 and $4 \mathrm{~h}$ were similar with pain/discomfort scores $\geq 3$ vs $<3$. Opiates and antiemetics were used by higher percentages with pain/discomfort scores $\geq 3$ (both $P<0.05$ ). Other factors were not different in the severity subgroups.

\section{Subgroup analysis by etiology}

Separate analyses assessed differences in diabetic vs idiopathic patients. Eighty-four $(61 \%)$ and $88(64 \%)$ diabetics reported moderate or greater upper pain and discomfort, respectively (Fig. 1B). Similar pain/discomfort distributions were observed for idiopathic patients (Fig. 1C). Factors exhibiting increases in diabetics with pain scores $\geq 3$ included overall GCSI scores and nausea/vomiting, fullness/early satiety, and bloating/ distention subscale scores; PAGI-SYM lower pain and discomfort and constipation scores; overall BPI scores and subscores for general activity, mood, relations with people, sleep, and enjoyment of life; BDI and STAI Y1 and Y2 scores; and opiate use (all $P \leq 0.013$ )(Table 2). Clinician-rated severity was greater, and PAGI-QOL and SF-36 physical and mental component scores were more impaired in diabetics in the greater severity subgroup (all $P \leq 0.001$ ). Prevalences of neuropathy and hemoglobin Alc values were similar in diabetics with pain scores $\geq 3$ vs $<3$.

Factors relating to pain/discomfort severity in idiopathic gastroparesis were similar to diabetics; higher overall GCSI scores and nausea/vomiting, fullness/ early satiety, and bloating/distention subscale scores; PAGI-SYM lower abdominal pain/discomfort, constipation, and diarrhea scores; BPI subscores and overall pain interference; and BDI and STAI Y1 and Y2 scores were higher in the greater severity subset (all $P \leq 0.004)($ Table 2). Impairments of PAGI-QOL and SF-36 physical and mental components were noted in idiopathics with higher pain/discomfort scores (all $P \leq 0.001)$.

\section{Abdominal pain/discomfort- vs. nausea/vomiting predominance}

Of 393 patients, $81(21 \%)$ reported abdominal pain/ discomfort predominance; most reported either predominant upper pain or pain location not specified, with few reporting predominant lower pain or discomfort in any location (Table 3). Predominant nausea/ vomiting was declared by $172(44 \%)$; most reported predominant nausea with few reporting predominant vomiting and one patient noting predominant retching. Other symptoms were predominant in 139 (35\%); one patient did not declare symptom predominance.

Factors were compared with predominant pain/discomfort vs nausea/vomiting (Table 4). PAGI-SYM upper pain and discomfort scores, BPI subscores for general activity, walking ability, and enjoyment of life, 
Table 1 Relation of upper abdominal pain/upper abdominal discomfort to baseline characteristics of gastroparesis patients

\begin{tabular}{|c|c|c|c|c|c|}
\hline Characteristic & & $\begin{array}{l}\text { Upper abdominal pain/ } \\
\text { Upper abdominal } \\
\text { discomfort score } \geq 3 \\
\mathrm{~N}=288\end{array}$ & $\begin{array}{l}\text { Upper abdominal pain/ } \\
\text { Upper abdominal } \\
\text { discomfort score }<3 \\
\mathrm{~N}=105\end{array}$ & Difference $(95 \% \mathrm{CI})^{\star}$ & $P$ \\
\hline \multirow{12}{*}{$\begin{array}{l}\text { Demographic } \\
\text { and clinical } \\
\text { factors }\end{array}$} & Age at enrollment (years) & $42.1 \pm 13.5$ & $43.6 \pm 14.7$ & $-1.5(-4.6,1.6)$ & 0.35 \\
\hline & Female sex & $246(85 \%)$ & $81(77 \%)$ & $8 \%(-1 \%, 18 \%)$ & 0.052 \\
\hline & Hispanic ethnicity & $14(5 \%)$ & $5(5 \%)$ & $0 \%(-6 \%, 5 \%)$ & 1.00 \\
\hline & Race & & & & \\
\hline & White & $249(87 \%)$ & $88(85 \%)$ & $2 \%(-7 \%, 10 \%)$ & 0.82 \\
\hline & Black & $27(9 \%)$ & $11(11 \%)$ & $-2 \%(-9 \%, 6 \%)$ & \\
\hline & Other & $9(3 \%)$ & $4(4 \%)$ & $-1 \%(-6 \%, 4 \%)$ & \\
\hline & Gastroparesis etiology: & & & & \\
\hline & Diabetic & $92(32 \%)$ & $45(43 \%)$ & $-11 \%(-22 \%, 1 \%)$ & 0.045 \\
\hline & Idiopathic & $196(68 \%)$ & $60(57 \%)$ & $11 \%(-1 \%, 22 \%)$ & \\
\hline & Acute symptom onset & $145(51 \%)$ & $58(56 \%)$ & $-5 \%(-17 \%, 7 \%)$ & 0.36 \\
\hline & $\begin{array}{l}\text { Initial infectious } \\
\text { prodrome }\end{array}$ & $44(15 \%)$ & $23(22 \%)$ & $-7 \%(-16 \%, 3 \%)$ & 0.122 \\
\hline \multirow{13}{*}{$\begin{array}{l}\text { Gastroparesis } \\
\text { severity } \\
\text { measures }\end{array}$} & Clinician-rated severity: & & & & \\
\hline & Grade 1 & $27(9 \%)$ & $22(21 \%)$ & $-12 \%(-21 \%,-3 \%)$ & 0.008 \\
\hline & Grade 2 & $156(55 \%)$ & $52(50 \%)$ & $5 \%(-7 \%, 16 \%)$ & \\
\hline & Grade 3 & $103(36 \%)$ & $30(29 \%)$ & $7 \%(-4 \%, 18 \%)$ & \\
\hline & GCSI: & & & & \\
\hline & Overall & $3.3 \pm 0.9$ & $2.2 \pm 1.0$ & $1.1(0.9,1.3)$ & $<0.001$ \\
\hline & Nausea/vomiting subscale & $2.8 \pm 1.3$ & $1.9 \pm 1.5$ & $0.9(0.5,1.2)$ & $<0.001$ \\
\hline & $\begin{array}{l}\text { Postprandial fullness/ } \\
\text { early satiety subscale }\end{array}$ & $3.7 \pm 0.9$ & $2.5 \pm 1.1$ & $1.2(1.0,1.4)$ & $<0.001$ \\
\hline & $\begin{array}{l}\text { Bloating subscale } \\
\text { PAGI-SYM: }\end{array}$ & $3.3 \pm 1.5$ & $2.0 \pm 1.5$ & $1.3(1.0,1.6)$ & $<0.001$ \\
\hline & Lower abdominal pain & $2.5 \pm 1.6$ & $1.2 \pm 1.4$ & $1.3(1.0,1.7)$ & $<0.001$ \\
\hline & $\begin{array}{l}\text { Lower abdominal } \\
\text { discomfort }\end{array}$ & $2.6 \pm 1.6$ & $1.4 \pm 1.5$ & $1.2(0.8,1.5)$ & $<0.001$ \\
\hline & Constipation & $2.8 \pm 1.7$ & $1.5 \pm 1.6$ & $1.2(0.8,1.6)$ & $<0.001$ \\
\hline & Diarrhea & $2.1 \pm 1.7$ & $1.4 \pm 1.5$ & $0.6(0.2,1.0)$ & 0.001 \\
\hline \multirow{12}{*}{$\begin{array}{l}\text { Quality of } \\
\text { life } \\
\text { measures }\end{array}$} & $\begin{array}{l}\text { PAGI-QOL } \\
S F-36:\end{array}$ & $2.1 \pm 1.0$ & $3.2 \pm 0.9$ & $-1.1(-1.3,-0.9)$ & $<0.001$ \\
\hline & Physical component & $31.0 \pm 9.1$ & $38.5 \pm 10.7$ & $-7.5(-9.7,-5.4)$ & $<0.0011$ \\
\hline & Mental component & $34.8 \pm 12.1$ & $42.3 \pm 12.3$ & $-7.4(-10.2,-4.7)$ & $<0.00$ \\
\hline & BPI: & & & & \\
\hline & $\begin{array}{l}\text { Overall pain } \\
\text { interference score }\end{array}$ & $5.9 \pm 2.5$ & $3.7 \pm 2.7$ & $2.2(1.5,2.9)$ & $<0.001$ \\
\hline & General activity & $6.0 \pm 3.0$ & $3.6 \pm 3.2$ & $2.3(1.5,3.2)$ & $<0.001$ \\
\hline & Mood & $6.3 \pm 2.9$ & $3.9 \pm 3.1$ & $2.4(1.6,3.2)$ & $<0.001$ \\
\hline & Walking ability & $4.7 \pm 3.2$ & $3.0 \pm 3.2$ & $1.7(0.8,2.6)$ & $<0.001$ \\
\hline & Normal work & $6.3 \pm 3.1$ & $4.4 \pm 3.4$ & $1.9(1.0,2.8)$ & $<0.001$ \\
\hline & Relations with people & $5.1 \pm 3.2$ & $3.2 \pm 3.1$ & $1.8(0.9,2.7)$ & $<0.001$ \\
\hline & Sleep & $6.2 \pm 3.1$ & $3.6 \pm 3.4$ & $2.6(1.7,3.5)$ & $<0.001$ \\
\hline & Enjoyment of life & $6.5 \pm 3.1$ & $4.2 \pm 3.3$ & $2.3(1.4,3.2)$ & $<0.001$ \\
\hline \multirow{4}{*}{$\begin{array}{l}\text { Parameters of } \\
\text { psychological } \\
\text { dysfunction }\end{array}$} & BDI & $21.2 \pm 11.3$ & $14.3 \pm 9.3$ & $6.9(4.5,9.3)$ & $<0.001$ \\
\hline & STAI: & & & & \\
\hline & Y1 state anxiety & $47.9 \pm 13.4$ & $39.6 \pm 11.8$ & $8.3(5.3,11.2)$ & $<0.001$ \\
\hline & $\mathrm{Y} 2$ trait anxiety & $46.4 \pm 12.3$ & $39.7 \pm 11.5$ & $6.7(4.0,9.4)$ & $<0.001$ \\
\hline \multirow{3}{*}{$\begin{array}{l}\text { Gastric } \\
\text { emptying }\end{array}$} & Gastric retention(\%): & & & & \\
\hline & $2 \mathrm{~h}$ & $64.3 \pm 18.2$ & $64.4 \pm 19.1$ & $-0.1(-4.2,4.1)$ & 0.97 \\
\hline & $4 \mathrm{~h}$ & $32.7 \pm 22.3$ & $32.7 \pm 23.5$ & $0.1(-5.0,5.1)$ & 0.98 \\
\hline \multirow{5}{*}{$\begin{array}{l}\text { Medication } \\
\text { use }\end{array}$} & Prokinetics & $157(54 \%)$ & $61(58 \%)$ & $-4 \%(-15 \%, 8 \%)$ & 0.53 \\
\hline & Antiemetics & $191(66 \%)$ & $58(55 \%)$ & $11 \%(-1 \%, 23 \%)$ & 0.044 \\
\hline & Opiates & $138(48 \%)$ & $35(33 \%)$ & $15 \%(3 \%, 26 \%)$ & 0.009 \\
\hline & Neuropathic pain modulators & $59(20 \%)$ & $19(18 \%)$ & $2 \%(-7 \%, 12 \%)$ & 0.60 \\
\hline & Antidepressants & $109(38 \%)$ & $34(32 \%)$ & $6 \%(-6 \%, 17 \%)$ & 0.32 \\
\hline
\end{tabular}

All data are mean $\pm \mathrm{SD}$ or $\mathrm{N}(\%)$.

${ }^{\star}$ Differences in means $(95 \% \mathrm{CI})$ are presented for continuous variables and differences in proportions $(95 \% \mathrm{CI})$ are presented for categorical variables. The $95 \%$ CIs for differences in means were based on the $t$-distribution; intervals for differences in proportions used the Newcombe score method with continuity correction.

GCSI, Gastroparesis Cardinal Symptom Index; PAGI-SYM, Patient Assessment of Upper Gastrointestinal Disorders Symptoms; PAGI-QOL, Patient Assessment of Upper Gastrointestinal Disorders Quality of Life; SF-36, Short Form-36; BPI, Brief Pain Inventory; BDI, Beck Depression Inventory; STAI, State and Trait Anxiety Inventory. 
Table 2 Relation of upper abdominal pain/upper abdominal discomfort to baseline characteristics in diabetic and idiopathic gastroparesis

\begin{tabular}{|c|c|c|c|c|c|c|c|}
\hline \multirow[b]{2}{*}{ Characteristic } & & \multicolumn{3}{|c|}{ Diabetic gastroparesis } & \multicolumn{3}{|l|}{ Idiopathic gastroparesis } \\
\hline & & $\begin{array}{l}\text { Upper abdominal } \\
\text { pain/Upper } \\
\text { abdominal } \\
\text { discomfort } \\
\text { score } \geq 3 \mathrm{~N}=92\end{array}$ & $\begin{array}{l}\text { Upper abdominal } \\
\text { pain/Upper } \\
\text { abdominal } \\
\text { discomfort } \\
\text { score }<3 \mathrm{~N}=45\end{array}$ & $P$ & $\begin{array}{l}\text { Upper abdominal } \\
\text { pain/Upper abdominal } \\
\text { discomfort score } \geq 3 \\
\mathrm{~N}=196\end{array}$ & $\begin{array}{l}\text { Upper abdominal } \\
\text { pain/Upper } \\
\text { abdominal } \\
\text { discomfort } \\
\text { score }<3 \\
\mathrm{~N}=60\end{array}$ & $P$ \\
\hline \multirow{9}{*}{$\begin{array}{l}\text { Demographic } \\
\text { and clinical } \\
\text { factors }\end{array}$} & $\begin{array}{l}\text { Age at enrollment } \\
\text { (years) }\end{array}$ & $43.8 \pm 13.2$ & $47.5 \pm 12.4$ & 0.112 & $41.3 \pm 13.6$ & $40.6 \pm 15.7$ & 0.73 \\
\hline & Female sex & $70(76 \%)$ & $30(67 \%)$ & 0.31 & $176(90 \%)$ & $51(85 \%)$ & 0.35 \\
\hline & $\begin{array}{l}\text { Hispanic } \\
\text { ethnicity }\end{array}$ & $8(9 \%)$ & $4(9 \%)$ & 1.00 & $6(3 \%)$ & $1(2 \%)$ & 1.00 \\
\hline & Race: & & & & & & \\
\hline & White & $69(76 \%)$ & $36(84 \%)$ & 0.47 & $180(93 \%)$ & $52(87 \%)$ & 0.20 \\
\hline & Black & $18(20 \%)$ & $5(12 \%)$ & & $9(5 \%)$ & $6(10 \%)$ & \\
\hline & Other & $4(4 \%)$ & $2(5 \%)$ & & $5(3 \%)$ & $2(3 \%)$ & \\
\hline & $\begin{array}{l}\text { Acute } \\
\text { symptom } \\
\text { onset }\end{array}$ & $45(50 \%)$ & $28(64 \%)$ & 0.143 & $100(51 \%)$ & $30(50 \%)$ & 0.89 \\
\hline & $\begin{array}{l}\text { Initial } \\
\text { infectious } \\
\text { prodrome }\end{array}$ & $11(12 \%)$ & $8(18 \%)$ & 0.43 & $33(17 \%)$ & $15(25 \%)$ & 0.189 \\
\hline \multirow{13}{*}{$\begin{array}{l}\text { Gastroparesis } \\
\text { severity } \\
\text { measures }\end{array}$} & \multicolumn{7}{|l|}{ Clinician-rated severity: } \\
\hline & Grade 1 & $2(2 \%)$ & $10(23 \%)$ & $<0.001$ & $25(13 \%)$ & $12(20 \%)$ & 0.24 \\
\hline & Grade 2 & $46(50 \%)$ & $17(39 \%)$ & & $110(57 \%)$ & $35(58 \%)$ & \\
\hline & Grade 3 & $44(48 \%)$ & $17(39 \%)$ & & $59(30 \%)$ & $13(22 \%)$ & \\
\hline & \multicolumn{7}{|l|}{ GCSI: } \\
\hline & Overall & $3.3 \pm 0.8$ & $2.2 \pm 1.0$ & $<0.001$ & $3.3 \pm 0.9$ & $2.1 \pm 0.9$ & $<0.001$ \\
\hline & $\begin{array}{l}\text { Nausea/vomiting } \\
\text { subscale }\end{array}$ & $3.1 \pm 1.3$ & $2.2 \pm 1.6$ & $<0.001$ & $2.6 \pm 1.4$ & $1.7 \pm 1.4$ & $<0.001$ \\
\hline & $\begin{array}{l}\text { Fullness/satiety } \\
\text { subscale }\end{array}$ & $3.5 \pm 0.9$ & $2.4 \pm 1.2$ & $<0.001$ & $3.8 \pm 0.9$ & $2.6 \pm 1.1$ & $<0.001$ \\
\hline & $\begin{array}{l}\text { Bloating subscale } \\
\text { PAGI-SYM: }\end{array}$ & $3.3 \pm 1.5$ & $2.0 \pm 1.5$ & $<0.001$ & $3.4 \pm 1.5$ & $2.1 \pm 1.5$ & $<0.001$ \\
\hline & $\begin{array}{l}\text { Lower abdominal } \\
\text { pain }\end{array}$ & $2.6 \pm 1.5$ & $0.9 \pm 1.1$ & $<0.001$ & $2.4 \pm 1.6$ & $1.4 \pm 1.5$ & $<0.001$ \\
\hline & Lower discomfort & $2.7 \pm 1.5$ & $1.2 \pm 1.2$ & $<0.001$ & $2.5 \pm 1.7$ & $1.6 \pm 1.6$ & $<0.001$ \\
\hline & Constipation & $2.7 \pm 1.6$ & $1.6 \pm 1.6$ & $<0.001$ & $2.8 \pm 1.8$ & $1.5 \pm 1.6$ & $<0.001$ \\
\hline & Diarrhea & $2.1 \pm 1.8$ & $1.6 \pm 1.5$ & 0.129 & $2.1 \pm 1.7$ & $1.3 \pm 1.5$ & 0.003 \\
\hline \multirow{12}{*}{$\begin{array}{l}\text { Quality of } \\
\text { life } \\
\text { measures }\end{array}$} & $\begin{array}{l}\text { PAGI-QOL } \\
\text { SF-36: }\end{array}$ & $2.1 \pm 1.1$ & $3.2 \pm 1.0$ & $<0.001$ & $2.2 \pm 1.0$ & $3.3 \pm 0.9$ & $<0.001$ \\
\hline & $\begin{array}{l}\text { Physical } \\
\text { component }\end{array}$ & $29.0 \pm 9.0$ & $35.9 \pm 9.6$ & $<0.001$ & $31.9 \pm 9.1$ & $40.5 \pm 11.1$ & $<0.001$ \\
\hline & $\begin{array}{l}\text { Mental } \\
\text { component }\end{array}$ & $32.6 \pm 11.7$ & $41.5 \pm 12.7$ & $<0.001$ & $35.8 \pm 12.2$ & $42.9 \pm 12.0$ & $<0.001$ \\
\hline & BPI: & & & & & & \\
\hline & $\begin{array}{l}\text { Pain interference } \\
\text { score }\end{array}$ & $6.2 \pm 2.4$ & $4.4 \pm 2.8$ & 0.003 & $5.7 \pm 2.5$ & $3.3 \pm 2.6$ & $<0.001$ \\
\hline & General activity & $6.3 \pm 2.7$ & $4.1 \pm 3.6$ & 0.001 & $5.8 \pm 3.1$ & $3.4 \pm 3.0$ & $<0.001$ \\
\hline & Mood & $6.6 \pm 2.7$ & $4.2 \pm 3.4$ & $<0.001$ & $6.2 \pm 2.9$ & $3.7 \pm 2.9$ & $<0.001$ \\
\hline & Walking ability & $5.2 \pm 3.0$ & $4.5 \pm 3.2$ & 0.31 & $4.5 \pm 3.2$ & $2.1 \pm 2.8$ & $<0.001$ \\
\hline & Normal work & $6.7 \pm 2.7$ & $5.8 \pm 3.3$ & 0.185 & $6.1 \pm 3.2$ & $3.5 \pm 3.3$ & $<0.001$ \\
\hline & $\begin{array}{l}\text { Relations with } \\
\text { people }\end{array}$ & $5.4 \pm 3.1$ & $3.3 \pm 3.2$ & 0.004 & $5.0 \pm 3.3$ & $3.2 \pm 3.0$ & 0.004 \\
\hline & Sleep & $6.3 \pm 3.1$ & $4.4 \pm 3.9$ & 0.013 & $6.2 \pm 3.2$ & $3.1 \pm 3.0$ & $<0.001$ \\
\hline & Enjoyment of life & $6.8 \pm 3.1$ & $4.8 \pm 3.3$ & 0.006 & $6.4 \pm 3.1$ & $3.9 \pm 3.2$ & $<0.001$ \\
\hline \multirow{3}{*}{$\begin{array}{l}\text { Parameters of } \\
\text { psychological } \\
\text { dysfunction }\end{array}$} & $\begin{array}{l}\text { BDI } \\
\text { STAI: }\end{array}$ & $22.8 \pm 11.9$ & $15.0 \pm 9.7$ & $<0.001$ & $20.4 \pm 11.0$ & $13.7 \pm 9.1$ & $<0.001$ \\
\hline & Y1 state anxiety & $49.9 \pm 13.0$ & $39.1 \pm 12.2$ & $<0.001$ & $46.9 \pm 13.5$ & $40.0 \pm 11.7$ & $<0.001$ \\
\hline & $\mathrm{Y} 2$ trait anxiety & $48.5 \pm 12.3$ & $40.2 \pm 12.4$ & $<0.001$ & $45.4 \pm 12.2$ & $39.3 \pm 10.9$ & $<0.001$ \\
\hline \multirow{3}{*}{$\begin{array}{l}\text { Gastric } \\
\text { emptying }\end{array}$} & Gastric retention (\%): & & & & & & \\
\hline & $2 \mathrm{~h}$ & $67.4 \pm 21.7$ & $66.0 \pm 20.5$ & 0.71 & $62.9 \pm 16.1$ & $63.3 \pm 18.0$ & 0.89 \\
\hline & $4 \mathrm{~h}$ & $40.3 \pm 26.5$ & $41.7 \pm 27.0$ & 0.77 & $29.2 \pm 19.0$ & $25.9 \pm 18.0$ & 0.24 \\
\hline
\end{tabular}


Table 2 (Continued)

\begin{tabular}{|c|c|c|c|c|c|c|c|}
\hline \multirow[b]{2}{*}{ Characteristic } & & \multicolumn{3}{|c|}{ Diabetic gastroparesis } & \multicolumn{3}{|l|}{ Idiopathic gastroparesis } \\
\hline & & $\begin{array}{l}\text { Upper abdominal } \\
\text { pain/Upper } \\
\text { abdominal } \\
\text { discomfort } \\
\text { score } \geq 3 \mathrm{~N}=92\end{array}$ & $\begin{array}{l}\text { Upper abdominal } \\
\text { pain/Upper } \\
\text { abdominal } \\
\text { discomfort } \\
\text { score }<3 \mathrm{~N}=45\end{array}$ & $P$ & $\begin{array}{l}\text { Upper abdominal } \\
\text { pain/Upper abdominal } \\
\text { discomfort score } \geq 3 \\
\mathrm{~N}=196\end{array}$ & $\begin{array}{l}\text { Upper abdominal } \\
\text { pain/Upper } \\
\text { abdominal } \\
\text { discomfort } \\
\text { score }<3 \\
\mathrm{~N}=60\end{array}$ & $P$ \\
\hline \multirow{5}{*}{$\begin{array}{l}\text { Medication } \\
\text { use }\end{array}$} & Prokinetics & $64(70 \%)$ & $28(62 \%)$ & 0.44 & $93(48 \%)$ & $33(55 \%)$ & 0.31 \\
\hline & Antiemetics & $66(72 \%)$ & $28(62 \%)$ & 0.33 & $125(64 \%)$ & $30(50 \%)$ & 0.056 \\
\hline & Opiates & $49(53 \%)$ & $15(33 \%)$ & 0.031 & $89(46 \%)$ & $20(33 \%)$ & 0.092 \\
\hline & Pain modulators & $24(26 \%)$ & $13(29 \%)$ & 0.84 & $35(18 \%)$ & $6(10 \%)$ & 0.164 \\
\hline & Antidepressants & $37(40 \%)$ & $15(33 \%)$ & 0.46 & $72(37 \%)$ & $19(32 \%)$ & 0.54 \\
\hline \multirow{2}{*}{$\begin{array}{l}\text { Diabetes-related } \\
\text { factors }\end{array}$} & Neuropathy & 35 (38\%) & $21(47 \%)$ & 0.33 & & & \\
\hline & Hemoglobin Alc (\%) & $7.9 \pm 2.0$ & $7.9 \pm 2.0$ & 0.99 & & & \\
\hline
\end{tabular}

All data are mean $\pm \mathrm{SD}$ or $\mathrm{N}(\%)$.

GCSI, Gastroparesis Cardinal Symptom Index; PAGI-SYM, Patient Assessment of Upper Gastrointestinal Disorders Symptoms; PAGI-QOL, Patient Assessment of Upper Gastrointestinal Disorders Quality of Life; SF-36, Short Form-36; BPI, Brief Pain Inventory; BDI, Beck Depression Inventory; STAI, State and Trait Anxiety Inventory.

Table 3 Patient-reported symptom predominance from the PAGI-SYM

\begin{tabular}{|c|c|c|c|c|c|}
\hline $\begin{array}{l}\text { Predominant symptom } \\
\text { subscale }\end{array}$ & Predominant symptom & $\begin{array}{l}\text { Individual } \\
\text { symptom } \mathrm{N}\end{array}$ & $\begin{array}{l}\text { Individual } \\
\text { symptom \% }\end{array}$ & $\begin{array}{l}\text { Symptom } \\
\text { subscale N }\end{array}$ & $\begin{array}{l}\text { Symptom } \\
\text { subscale \% }\end{array}$ \\
\hline \multirow{6}{*}{$\begin{array}{l}\text { Abdominal pain/ } \\
\text { discomfort }\end{array}$} & Upper abdominal pain & 27 & $7 \%$ & \multirow[t]{6}{*}{81} & \multirow[t]{6}{*}{$21 \%$} \\
\hline & Abdominal pain location not specified & 42 & $11 \%$ & & \\
\hline & Lower abdominal pain & 6 & $2 \%$ & & \\
\hline & Upper abdominal discomfort & 2 & $0.5 \%$ & & \\
\hline & Abdominal discomfort location not specified & 2 & $0.5 \%$ & & \\
\hline & Lower abdominal discomfort & 2 & $0.5 \%$ & & \\
\hline \multirow{3}{*}{ Nausea/vomiting } & Nausea & 135 & $34 \%$ & \multirow[t]{3}{*}{172} & \multirow[t]{3}{*}{$44 \%$} \\
\hline & Vomiting & 36 & $9 \%$ & & \\
\hline & Retching & 1 & $0.3 \%$ & & \\
\hline \multirow{4}{*}{$\begin{array}{l}\text { Postprandial } \\
\text { fullness/ } \\
\text { early satiety }\end{array}$} & Stomach fullness & 26 & $7 \%$ & \multirow[t]{4}{*}{47} & \multirow[t]{4}{*}{$12 \%$} \\
\hline & Not able to finish normal-sized meal & 8 & $2 \%$ & & \\
\hline & Feeling excessively full after meals & 8 & $2 \%$ & & \\
\hline & Loss of appetite & 5 & $1 \%$ & & \\
\hline \multirow{2}{*}{$\begin{array}{l}\text { Bloating/ } \\
\text { distention }\end{array}$} & Bloating & 26 & $7 \%$ & \multirow[t]{2}{*}{27} & \multirow[t]{2}{*}{$7 \%$} \\
\hline & Stomach or belly visibly larger & 1 & $0.3 \%$ & & \\
\hline \multirow{8}{*}{$\begin{array}{l}\text { Esophageal } \\
\text { symptoms }\end{array}$} & Heartburn during the day & 1 & $0.3 \%$ & \multirow[t]{8}{*}{32} & \multirow[t]{8}{*}{$8 \%$} \\
\hline & Heartburn, did not specify day $v S$ when lying down & 5 & $1 \%$ & & \\
\hline & Feeling of discomfort inside chest during day & 1 & $0.3 \%$ & & \\
\hline & $\begin{array}{l}\text { Feeling of discomfort inside chest, did not specify } \\
\text { day vs lying down }\end{array}$ & 4 & $1 \%$ & & \\
\hline & Regurgitation or reflux during day & 4 & $1 \%$ & & \\
\hline & Regurgitation or reflux lying down & 3 & $1 \%$ & & \\
\hline & $\begin{array}{l}\text { Regurgitation or reflux, did not specify day vs. } \\
\text { lying down }\end{array}$ & 12 & $3 \%$ & & \\
\hline & Bitter, acid or sour taste in mouth & 2 & $0.5 \%$ & & \\
\hline \multirow{3}{*}{$\begin{array}{l}\text { Bowel habit } \\
\text { abnormalities }\end{array}$} & Constipation & 18 & $5 \%$ & \multirow[t]{2}{*}{33} & \multirow[t]{2}{*}{$8 \%$} \\
\hline & Diarrhea & 15 & $4 \%$ & & \\
\hline & No symptom specified & 1 & $0.3 \%$ & 1 & $0.3 \%$ \\
\hline
\end{tabular}

and overall pain interference scores were higher with predominant pain/discomfort, while overall GCSI and nausea/vomiting subscale scores were higher with predominant nausea/vomiting (all $P \leq 0.039$ ). Opiates were used by greater percentages with predominant pain/discomfort, while antiemetics were taken by larger percentages with predominant nausea/vomiting (both $P \leq 0.002$ ). There were no differences in other factors between predominance subgroups.

\section{Multiple logistic regression analyses}

Multiple logistic regression analysis identified characteristics independently associated with upper abdom- 
Table 4 Characteristics of gastroparesis patients with predominant pain/discomfort vs predominant nausea/vomiting

\begin{tabular}{|c|c|c|c|c|c|}
\hline Characteristic & & $\begin{array}{l}\text { Predominant abdominal } \\
\text { pain/Discomfort } N=81\end{array}$ & $\begin{array}{l}\text { Predominant } \\
\text { nausea/Vomiting } \\
\mathrm{N}=172\end{array}$ & Difference $(95 \% \mathrm{CI})^{\star}$ & $P$ \\
\hline \multirow{12}{*}{$\begin{array}{l}\text { Demographic and } \\
\text { clinical factors }\end{array}$} & Age at enrollment (years) & $41.3 \pm 12.9$ & $40.4 \pm 14.2$ & $0.9(-2.8,4.6)$ & 0.63 \\
\hline & Female sex & $68(84 \%)$ & $144(84 \%)$ & $0 \%(-10 \%, 11 \%)$ & 1.00 \\
\hline & Hispanic ethnicity & $4(5 \%)$ & $8(5 \%)$ & $0 \%(-6 \%, 7 \%)$ & 1.00 \\
\hline & Race: & & & & \\
\hline & White & $66(84 \%)$ & $149(88 \%)$ & $-4 \%(-15 \%, 6 \%)$ & 0.55 \\
\hline & Black & $11(14 \%)$ & $16(10 \%)$ & $4 \%(-5 \%, 14 \%)$ & \\
\hline & Other & $2(2 \%)$ & $4(2 \%)$ & $0 \%(-5 \%, 5 \%)$ & \\
\hline & Gastroparesis etiology: & & & & \\
\hline & Diabetic & $23(28 \%)$ & $57(33 \%)$ & $-5 \%(-18 \%, 8 \%)$ & 0.45 \\
\hline & Idiopathic & $58(72 \%)$ & $115(67 \%)$ & $5 \%(-8 \%, 18 \%)$ & \\
\hline & Acute symptom onset & $42(52 \%)$ & $100(58 \%)$ & $-6 \%(-20 \%, 8 \%)$ & 0.40 \\
\hline & Initial infectious prodrome & $12(15 \%)$ & $32(19 \%)$ & $-4 \%(-14 \%, 7 \%)$ & 0.46 \\
\hline \multirow{16}{*}{$\begin{array}{l}\text { Gastroparesis } \\
\text { severity } \\
\text { measures }\end{array}$} & Clinician-rated severity: & & & & \\
\hline & Grade 1 & $7(9 \%)$ & $15(9 \%)$ & $0 \%(-9 \%, 8 \%)$ & 0.90 \\
\hline & Grade 2 & $39(49 \%)$ & $88(52 \%)$ & $-3 \%(-17 \%, 11 \%)$ & \\
\hline & Grade 3 & $34(42 \%)$ & $67(39 \%)$ & $3 \%(-11 \%, 17 \%)$ & \\
\hline & GCSI: & & & & \\
\hline & Overall & $2.9 \pm 1.0$ & $3.2 \pm 0.9$ & $-0.4(-0.6,-0.1)$ & 0.003 \\
\hline & Nausea/vomiting subscale & $2.3 \pm 1.3$ & $3.3 \pm 1.3$ & $-1.0(-1.3,-0.7)$ & $<0.001$ \\
\hline & $\begin{array}{l}\text { Postprandial fullness/early } \\
\text { satiety subscale }\end{array}$ & $3.5 \pm 1.1$ & $3.5 \pm 1.0$ & $0(-0.3,0.2)$ & 0.74 \\
\hline & Bloating subscale & $2.9 \pm 1.6$ & $2.9 \pm 1.6$ & $-0.1(-0.5,0.3)$ & 0.69 \\
\hline & $\begin{array}{l}\text { PAGT-SYM: } \\
\text { Unner abdominal nain }\end{array}$ & $41+11$ & $29+18$ & & \\
\hline & $\begin{array}{l}\text { Upper abdominal pain } \\
\text { Upper abdominal discomfort }\end{array}$ & $\begin{array}{l}4.1 \pm 1.1 \\
4.1 \pm 1.1\end{array}$ & $\begin{array}{l}2.9 \pm 1.8 \\
3.1+1.6\end{array}$ & & $<0.001$ \\
\hline & & & $3.1 \pm 1.6$ & $1.0(0.6,1.3)$ & $<0.001$ \\
\hline & & $2.5 \pm 1.6$ & $2.2 \pm 1.7$ & $0.4(-0.1,0.8)$ & 0.114 \\
\hline & & $2.5 \pm 1.1$ & $2.3 \pm 1.7$ & $0.3(-0.2,0.7)$ & 0.26 \\
\hline & Constipation & $2.5 \pm 1.6$ & $2.4 \pm 1.8$ & $0.1(-0.4,0.5)$ & 0.78 \\
\hline & Diarrhea & $1.7 \pm 1.6$ & $2.0 \pm 1.8$ & $-0.3(-0.8,0.1)$ & 0.159 \\
\hline \multirow[t]{12}{*}{$\begin{array}{l}\text { Quality of life } \\
\text { measures }\end{array}$} & $\begin{array}{l}\text { PAGI-QOL } \\
\text { SF-36: }\end{array}$ & $2.2 \pm 1.1$ & $2.4 \pm 1.1$ & $-0.2(-0.5,0.0)$ & 0.095 \\
\hline & Physical component & $31.6 \pm 8.3$ & $32.5 \pm 10.0$ & $-0.9(-3.4,1.6)$ & 0.49 \\
\hline & Mental component & $35.3 \pm 10.8$ & $36.0 \pm 12.9$ & $-0.7(-4.0,2.5)$ & 0.65 \\
\hline & BPI: & & & & \\
\hline & $\begin{array}{l}\text { Overall pain interference } \\
\text { score }\end{array}$ & $6.2 \pm 2.2$ & $5.4 \pm 2.8$ & $0.8(0.1,1.6)$ & 0.026 \\
\hline & General activity & $6.5 \pm 2.7$ & $5.5 \pm 3.3$ & $1.0(0.2,1.9)$ & 0.019 \\
\hline & Mood & $6.6 \pm 2.6$ & $5.9 \pm 3.3$ & $0.8(-0.1,1.7)$ & 0.067 \\
\hline & Walking ability & $5.1 \pm 2.9$ & $4.0 \pm 3.3$ & $1.0(0.2,1.9)$ & 0.022 \\
\hline & Normal work & $6.5 \pm 2.7$ & $6.1 \pm 3.4$ & $0.4(-0.5,1.3)$ & 0.37 \\
\hline & Relations with people & $5.4 \pm 3.1$ & $4.7 \pm 3.4$ & $0.7(-0.3,1.6)$ & 0.167 \\
\hline & Sleep & $6.5 \pm 3.2$ & $5.6 \pm 3.6$ & $0.9(-0.1,1.8)$ & 0.067 \\
\hline & Enjoyment of life & $7.0 \pm 2.9$ & $6.0 \pm 3.4$ & $1.0(0.0,1.9)$ & 0.039 \\
\hline \multirow{4}{*}{$\begin{array}{l}\text { Parameters of } \\
\text { psychological } \\
\text { dysfunction }\end{array}$} & BDI & $20.0 \pm 9.6$ & $19.9 \pm 11.2$ & $0.1(-2.8,2.9)$ & 0.98 \\
\hline & STAI: & & & & \\
\hline & Y1 state anxiety & $47.4 \pm 12.8$ & $46.5 \pm 13.5$ & $0.9(-2.6,4.4)$ & 0.62 \\
\hline & Y2 trait anxiety & $45.7 \pm 11.2$ & $45.1 \pm 12.6$ & $0.6(-2.6,3.8)$ & 0.71 \\
\hline Gastric & Gastric retention (\%): & & & & \\
\hline \multirow[t]{2}{*}{ emptying } & $2 \mathrm{~h}$ & $63.6 \pm 17.9$ & $63.5 \pm 17.7$ & $0.1(-4.6,4.8)$ & 0.97 \\
\hline & $4 \mathrm{~h}$ & $31.9 \pm 21.1$ & $32.3 \pm 21.9$ & $-0.5(-0.6,5.3)$ & 0.88 \\
\hline \multirow[t]{5}{*}{ Medication use } & Prokinetics & $43(53 \%)$ & $103(60 \%)$ & $-7 \%(-21 \%, 7 \%)$ & 0.31 \\
\hline & Antiemetics & $43(53 \%)$ & $130(76 \%)$ & $-22 \%(-36 \%,-9 \%)$ & $<0.001$ \\
\hline & Opiates & $49(60 \%)$ & $68(40 \%)$ & $20 \%(7 \%, 35 \%)$ & 0.002 \\
\hline & $\begin{array}{l}\text { Neuropathic pain } \\
\text { modulators }\end{array}$ & $18(22 \%)$ & $34(20 \%)$ & $2 \%(-9 \%, 14 \%)$ & 0.65 \\
\hline & Antidepressants & $34(42 \%)$ & $67(39 \%)$ & $3 \%(-11 \%, 17 \%)$ & 0.65 \\
\hline
\end{tabular}

All data are mean \pm SD or $\mathrm{N}(\%)$.

${ }^{\star}$ Differences in means $(95 \% \mathrm{CI})$ are presented for continuous variables and differences in proportions $(95 \% \mathrm{CI})$ are presented for categorical variables. The $95 \%$ CIs for differences in means were based on the t-distribution; intervals for differences in proportions used the Newcombe score method with continuity correction.

GCSI, Gastroparesis Cardinal Symptom Index; PAGI-SYM, Patient Assessment of Upper Gastrointestinal Disorders Symptoms; PAGI-QOL, Patient Assessment of Upper Gastrointestinal Disorders Quality of Life; SF-36, Short Form-36; BPI, Brief Pain Inventory; BDI, Beck Depression Inventory; STAI, State and Trait Anxiety Inventory. 
inal pain/discomfort scores of $\geq 3$ vs $<3$ and predominant pain/discomfort $v s$ predominant nausea/vomiting. Idiopathic etiology, higher overall GCSI scores, increased constipation severity, and impaired PAGIQOL and SF-36 physical component scores related to increased pain/discomfort severity (all $P<0.05$ ); initial infectious prodromes related to decreased severity scores $(P=0.017)($ Table $5 \mathrm{~A})$. Opiate use and impaired PAGI-QOL severity related to predominant pain/ discomfort $(P \leq 0.005)$; overall GCSI scores and antiemetic use related to predominant nausea/vomiting $(P<0.001)$ (Table 5B).

\section{DISCUSSION}

This study employed a large multicenter database with defined inclusion criteria, uniform validated surveys, and standardized scintigraphy methods to comprehensively characterize factors associated with moderatesevere pain in gastroparesis. Upper abdominal pain of

Table 5 Results of multiple logistic regression analyses. (A) characteristics independently associated with higher levels of upper abdominal pain/ discomfort (scores $\geq 3 \mathrm{vs}<3$ ), (B) Characteristics independently associated with predominant abdominal pain/discomfort vs predominant nausea/ vomiting

\begin{tabular}{|c|c|c|c|c|}
\hline Characteristic & & Odds ratio & $95 \%$ Confidence interval & $P$ \\
\hline \multicolumn{5}{|l|}{ A } \\
\hline \multirow[t]{3}{*}{ Demographic and clinical factors } & Idiopathic etiology & 2.16 & $1.08,4.31$ & 0.029 \\
\hline & Acute symptom onset & 1.56 & $0.79,3.08$ & 0.199 \\
\hline & Initial infectious prodrome & 0.35 & $0.15,0.83$ & 0.017 \\
\hline \multirow[t]{7}{*}{ Gastroparesis severity measures } & Clinician-rated severity: & & & \\
\hline & Grade 2 vs Grade 1 & 1.23 & $0.51,2.95$ & 0.29 \\
\hline & Grade 3 vs Grade 1 & 0.68 & $0.24,1.93$ & \\
\hline & Overall GCSI & 1.14 & $1.09,1.20$ & $<0.001$ \\
\hline & PAGI-SYM: & & & \\
\hline & Constipation & 1.44 & $1.16,1.77$ & 0.001 \\
\hline & Diarrhea & 1.06 & $0.86,1.30$ & 0.57 \\
\hline \multirow[t]{2}{*}{ Quality of life measures } & PAGI-QOL & 0.58 & $0.37,0.92$ & 0.021 \\
\hline & SF-36 Physical component & 0.96 & $0.93,1.00$ & 0.049 \\
\hline \multirow[t]{2}{*}{ Parameters of psychological dysfunction } & BDI & 0.98 & $0.94,1.03$ & 0.48 \\
\hline & STAI Y1 & 1.03 & $0.99,1.06$ & 0.142 \\
\hline Gastric emptying & Gastric retention at $2 \mathrm{~h}$ & 0.99 & $0.98,1.01$ & 0.39 \\
\hline \multirow[t]{5}{*}{ Medication use } & Prokinetics & 0.59 & $0.30,1.16$ & 0.124 \\
\hline & Antiemetics & 0.62 & $0.30,1.28$ & 0.196 \\
\hline & Opiates & 1.01 & $0.51,1.99$ & 0.99 \\
\hline & Neuropathic pain modulators & 1.22 & $0.52,2.87$ & 0.65 \\
\hline & Antidepressants & 1.36 & $0.71,2.61$ & 0.35 \\
\hline \multicolumn{5}{|c|}{ - } \\
\hline \multirow[t]{3}{*}{ Demographic and clinical factors } & Idiopathic etiology & 1.66 & $0.74,3.69$ & 0.22 \\
\hline & Acute symptom onset & 0.73 & $0.37,1.46$ & 0.37 \\
\hline & Initial infectious prodrome & 0.71 & $0.29,1.75$ & 0.46 \\
\hline \multirow[t]{7}{*}{ Gastroparesis severity measures } & Clinician-rated severity: & & & \\
\hline & Grade 2 vs Grade 1 & 1.03 & $0.32,3.33$ & 0.69 \\
\hline & Grade 3 vs Grade 1 & 1.42 & $0.40,5.08$ & \\
\hline & GCSI & 0.91 & $0.86,0.95$ & $<0.001$ \\
\hline & PAGI-SYM: & & & \\
\hline & Constipation & 1.03 & $0.83,1.29$ & 0.76 \\
\hline & Diarrhea & 0.91 & $0.74,1.12$ & 0.36 \\
\hline \multirow[t]{2}{*}{ Quality of life measures } & PAGI-QOL & 0.52 & $0.33,0.82$ & 0.005 \\
\hline & SF-36 - Physical component & 0.98 & $0.94,1.02$ & 0.28 \\
\hline \multirow[t]{2}{*}{ Parameters of psychological dysfunction } & BDI & 0.97 & $0.92,1.01$ & 0.160 \\
\hline & STAI - Y1 & 1.02 & $0.99,1.06$ & 0.27 \\
\hline Gastric emptying & Gastric retention at $2 \mathrm{~h}$ & 1.01 & $0.99,1.02$ & 0.54 \\
\hline \multirow[t]{5}{*}{ Quality of life measures } & Prokinetics & 0.60 & $0.30,1.21$ & 0.154 \\
\hline & Antiemetics & 0.21 & $0.09,0.48$ & $<0.001$ \\
\hline & Opiates & 3.38 & $1.61,7.10$ & 0.001 \\
\hline & Neuropathic pain modulators & 0.60 & $0.25,1.47$ & 0.27 \\
\hline & Antidepressants & 1.52 & $0.75,3.09$ & 0.25 \\
\hline
\end{tabular}

* Model adjusted for age, gender, and self-reported race and ethnicity.

GCSI, Gastroparesis Cardinal Symptom Index; PAGI-SYM, Patient Assessment of Upper Gastrointestinal Disorders Symptoms; PAGI-QOL, Patient Assessment of Upper Gastrointestinal Disorders Quality of Life; SF-36, Short Form-36;

Hosmer-Lemeshow Goodness-of-Fit $P=0.47$. 
at least moderate severity was reported by $66 \%$, related to idiopathic etiology, and approached significance relating to female sex. Pain correlated with overall gastroparesis severity and tracked with other upper and lower gastrointestinal symptom scores.

Factors associated with upper abdominal pain in gastroparesis patients were examined. Enteric infection triggers disease onset in some patients with functional disease. ${ }^{27-29}$ On logistic regression, infectious prodromes related to reduced pain severity suggesting differential symptom impact of enteric infection. We previously reported pronounced psychological dysfunction in gastroparesis. ${ }^{30}$ Pain correlated with impaired disease-specific and generic quality of life, worse depression, and state and trait anxiety, and several parameters of the Brief Pain Inventory which were similar in diabetic and idiopathic gastroparesis. These findings are similar to observations relating pain to psychological parameters in other chronic pain syndromes. In a functional gastroduodenal disease study, dyspepsia severity was determined by degrees of depression and somatization. ${ }^{31}$ Likewise in fibromyalgia patients, musculoskeletal pain severity related to depression scores. ${ }^{32}$ Neither 2 nor 4 h gastric retention correlated with pain/discomfort in gastroparetics of either etiology. We note that patients with normal gastric emptying were excluded from this investigation to restrict pain characterization to those with objectively confirmed gastroparesis to avoid the controversy of evaluating subjects more appropriately considered to have functional dyspepsia, even though symptom profiles in the conditions may overlap. ${ }^{17,27,33}$ Medications used for gastroparesis pain include tricyclic antidepressants, neuropathic pain modulators (gabapentin and pregabalin), and opiates. ${ }^{8,34,35}$ We observed greater opiate use (48\%) among patients with moderate to severe pain. Antiemetic use was greater with higher pain scores probably because pain severity tracked with nausea/vomiting. There were no differences in use of other drugs between severity subgroups. In diabetics, pain severity showed no relation to neuropathic complications or glycemic control.

Our novel comparison between pain/discomfort(21\% of patients) and nausea/vomiting-predominant $(44 \%)$ disease contrasted symptom influences on other factors in gastroparesis. Clinician-rated severity, depression, and state and trait anxiety scores were similar in pain/discomfort- vs nausea/vomiting predominant disease. Higher overall GCSI scores in the nausea/vomiting predominance group may relate to the lack of an abdominal pain score in the GCSI. ${ }^{20}$ Newer versions of the GCSI (e.g. GCSI daily diary) were unavailable during Gastroparesis Registry enroll- ment. ${ }^{36}$ Pain predominance was associated with greater impact on disease-specific quality of life. Gastric retention did not relate to symptom predominance. The predominant pain/discomfort subgroup reported higher opiate use $(60 \%$ vs $40 \%)$, while those with predominant nausea/vomiting more often took antiemetics. Characterizing a pain/discomfort predominant gastroparesis subgroup provides a foundation upon which controlled treatment trials can be conducted similar to symptom-specific therapies offered for different subtypes of functional disorders such as irritable bowel syndrome. Although speculative, it is conceivable that such characterizations of severe and/or predominant pain in gastroparesis might limit unnecessary diagnostic testing by clinicians searching for other causes of pain.

Our findings confirm some observations from small, prior series many of which determined pain in binary fashion, but conflict with others. Previous groups noted pain prevalences of $42 \%-90 \%$ vs nausea in $79 \%-93 \%$ of gastroparetics. ${ }^{3-8}$ Greater pain in idiopathic patients was seen in some series; but sex differences in pain were not described. ${ }^{3,4,7}$ Like our findings, pain scored lower in post viral idiopathic gastroparesis, pain severity correlated with impaired PAGI-QOL scores, and opiate use was prominent in other cohorts $(31 \%-50 \%))^{3,4,37}$ Older reports noted greater pain predominance $(44 \%-48 \%)$, sometimes approaching nausea/vomiting predominance $(51 \%))^{4,7}$ Others defined vomiting-, regurgitation-, and dyspepsia-predominant subtypes. ${ }^{38}$ Most single center studies show no relation or weak association of gastric emptying with pain. ${ }^{3,5-8,10-14}$ One group noted increased postprandial pain with delayed emptying selectively in idiopathic gastroparetics. ${ }^{12}$ Prior studies of gastroparesis pain used inconsistent or non-validated gastric emptying tests. Gastroparesis diagnoses were made clinically in $36 \%$ of patients who did not have delayed emptying in one report; emptying was not assessed in nearly $10 \%$ in another study. ${ }^{6,7}$

These exhaustive analyses had some limitations. We did not localize gastroparesis pain to a specific site and did not perform qualitative pain profiling. Prior smaller series have characterized pain localization (epigastric $36 \%-43 \%$ ), timing (postprandial $24 \%-80 \%$; nocturnal $80 \%$ ), frequency (daily $43 \%$; weekly $38 \%$; intermittent $24 \%-62 \%$ ), and character (burning, vague, crampy, sharp, pressure $16 \%-64 \%) .{ }^{3,6,7}$ Furthermore, qualitative and temporal relations of pain to other factors including bowel pattern were not assessed. Such questions were not posed as part of the extensive surveys administered to these patients. Pain predominance was determined by asking patients to write 
down which symptom was the predominant one. In contrast to pain severity which was reliably localized to the upper vs lower abdomen, many pain predominant patients did not restrict their pain to a specific anatomic site. Thus, symptoms in this subgroup cannot be definitively confined to the upper region. However, more than three times as many pain/discomfort predominant patients localized symptoms to the upper compared to the lower abdomen supporting that most of our findings relating to pain predominance likely are consequences of upper gut symptomatology.

Other issues may have contributed to our findings. Pain properties in this cohort may have been influenced by referral bias. Our patients were sent to principal investigators of the specialized motility centers of the Gastroparesis Consortium for advanced care from community providers or from other physicians within the study centers. Referrals were initiated to obtain second opinions for refractory symptoms or to gain access to prescription programs for domperidone, pyloric botulinum toxin injection, or gastric stimulator surgery. It is conceivable that those with prominent pain were selectively referred to our study centers because of a paucity of effective therapies for this gastroparesis symptom. Confounding effects of medications may have influenced which patients were included in these analyses by artificially delaying or accelerating gastric emptying. It was policy at each center to discontinue opiates for $72 \mathrm{~h}$ before scintigraphy. Effects of surreptitious narcotic use on emptying measures were minimal as gastric retention was identical with moderate-severe pain (with greater opiate use) vs milder pain (with less opiate use). Small numbers of patients were prescribed other medications that delay emptying including calcium channel antagonists, anticholinergics, and glucagon-like peptide-1 analogs, but it is unlikely these influenced pain reports as gastric retention did not relate to pain severity. Likewise, plasma glucose levels on the day of scintigraphy may have determined which diabetics were included in these analyses as readings $>270 \mathrm{mg} \mathrm{dL}^{-1}$ slightly prolong gastric retention. ${ }^{39}$ Some of the study centers do not perform finger stick glucose testing before emptying testing. As relations of pain severity to clinical factors were similar in gastroparetics of diabetic vs idiopathic etiology, it is probable that acute metabolic factors had limited impact on our findings.

Our findings suggest new avenues for gastroparesis research. Few patients with pain/discomfort predominance reported dominant discomfort and few in the nausea/vomiting predominant group declared retching as dominant, raising questions about the importance of these symptoms. Furthermore, patient definitions of discomfort may be nebulous; it is conceivable other symptoms including fullness or bloating could alternatively be reported as discomfort by some individuals. Next generation surveys may exclude these items, simplifying symptom assessments. Future questionnaire studies should also include qualitative characterizations of gastroparesis pain and its relation to other symptoms. Other than confirming a poor relation to gastric retention, limited insight into mechanisms of gastroparesis pain was provided by this study. As in functional dyspepsia, the importance of visceral hypersensitivity and impaired fundic accommodation in gastroparesis pain should be clarified in large cohorts. ${ }^{5,10,40,41}$ Likewise given the prevalence of small bowel and colon transit delays and associations of pain to bloating and bowel disturbances in gastroparesis, extragastric factors should be studied as well. ${ }^{42,43}$ Future investigations into the pathogenesis of gastroparesis pain may incorporate a range of physiologic studies including satiety testing (to screen for impaired intake capability), barostat measures (to discriminate impaired accommodation or heightened sensitivity), and wireless motility testing (to assess transit and pressure activity in other gut regions) as well as full thickness biopsy studies to correlate symptom reports and measures of gut dysfunction with histopathologic factors. ${ }^{44}$ Finally, pain reductions with traditional therapies of gastroparesis are inconsistently observed, however, no controlled trials have been designed with pain control as the primary outcome. ${ }^{6,17,33,45,46}$ The low rates of antidepressant and pain modulator use in this study may reflect either poor efficacy of such medications or infrequent prescription. Investigations of these drugs are warranted for pain-predominant gastroparesis.

In conclusion, moderate-severe upper abdominal pain is prevalent in gastroparesis and is associated with idiopathic etiology, lack of infectious prodrome, increases in other gastric and extragastric symptoms, and approaches significance to increased female sex. Conversely, pain severity does not relate to gastric retention, diabetic complications, or metabolic control. The impact of pain is evidenced by impaired quality of life, interference with activities, increased depression and anxiety, and opiate use. Pain/discomfort is predominant in one fifth of gastroparetics. Predominant pain has at least as great an impact on disease severity and quality of life as predominant nausea/vomiting.

\section{FUNDING}

This project received support from the National Institute of Diabetes and Digestive and Kidney Diseases (NIDDK) (grants 
U01DK073983, U01DK073975, U01DK073985, U01DK074007, U01DK073974, U01DK074008) as part of its funding of the Gastroparesis Clinical Research Consortium.

\section{DISCLOSURES}

None for all investigators.

\section{AUTHOR CONTRIBUTION}

Guarantor of Manuscript: William L. Hasler, MD Each Author's Contribution to Paper: William L. Hasler: Study design, subject recruitment, data collection, data analysis, manuscript preparation; Laura A. Wilson, Linda Lee, James Tonascia, Aynur UnalpArida: Study design, statistical analysis, manuscript preparation; Henry P. Parkman, Kenneth L. Koch, Thomas L. Abell, Linda Nguyen, Pankaj J. Pasricha, William J. Snape, Richard W. McCallum, Irene Sarosiek: Study design, subject recruitment, data collection, contribution to manuscript preparation; Gianrico Farrugia, Jorge Calles: Study design, contribution to manuscript preparation; Frank Hamilton: Study design, grant administration, contribution to manuscript preparation.

\section{REFERENCES}

1 Parkman HP, Hasler WL, Fisher RS. American Gastroenterological Association technical review on the diagnosis and treatment of gastroparesis. Gastroenterology 2004; 127: 1592-622.

2 Abell TL, Bernstein RK, Cutts T et al. Treatment of gastroparesis: a multidisciplinary clinical review. Neurogastroenterol Motil 2006; 18: 263-83.

3 Cherian D, Sachdeva P, Fisher RS, Parkman HP. Abdominal pain is a frequent symptom of gastroparesis. Clin Gastroenterol Hepatol 2010; 8: 676-81.

4 Soykan I, Sivri B, Sarosiek I, Kiernan B, McCallum RW. Demography, clinical characteristics, psychological and abuse profiles, treatment, and long-term follow-up of patients with gastroparesis. Dig Dis Sci 1998; 43: 2398-404.

5 Karamanolis G, Caenepeel P, Arts J, Tack J. Determinants of symptom pattern in idiopathic severely delayed gastric emptying: gastric emptying rate or proximal stomach dysfunction? Gut 2007; 56: 29-36.

6 Hoogerwerf WA, Pasricha PJ, Kalloo AN, Schuster MM. Pain: the overlooked symptom in gastroparesis. Am J Gastroenterol 1999; 94: 1029-33.

7 Bielefeldt K, Raza N, Zickmund SL. Different faces of gastroparesis. World I Gastroenterol 2009; 15: 6052-60.

8 Anaparthy R, Pehlivanov N, Grady J, Yimei H, Pasricha PJ. Gastroparesis and gastroparesis-like syndrome: response to therapy and its predictors. Dig Dis Sci 2009; 54: 1003-10.

9 Briley LC, Harrell SP, Woosley A, Eversmann J, Wo JM. National survey of physicians' perception of the cause, complications, and management of gastroparesis. South Med I 2011; 104: 412-7.
10 Kindt S, Dubois D, Van Oudenhove L et al. Relationship between symptom pattern, assessed by the PAGI-SYM questionnaire, and gastric sensorimotor dysfunction in functional dyspepsia. Neurogastroenterol Motil 2009; 21: 1183-e105.

11 Stanghellini V, Tosetti C, Horowitz $\mathrm{M}$ et al. Predictors of gastroparesis in out-patients with secondary and idiopathic upper gastrointestinal symptoms. Dig Liver Dis 2003; 35: 389-96.

12 Khayyam U, Sachdeva P, Gomez J et al. Assessment of symptoms during gastric emptying scintigraphy to correlate symptoms to delayed gastric emptying. Neurogastroenterol Motil 2010; 22: 539-45.

13 Jones KL, Wishart JM, Russo A, Wishart JM, Berry MK, Horowitz M. Predictors of delayed gastric emptying in diabetes. Diabetes Care 2001; 24: 1264-9.

14 Samsom M, Vermeijden JR, Smout AJ et al. Prevalence of delayed gastric emptying in diabetic patients and relationship to dyspeptic symptoms: a prospective study in unselected diabetic patients. Diabetes Care 2003; 26: 3116-22.

15 Silvers D, Kipnes M, Broadstone V et al. Domperidone in the management of symptoms of diabetic gastroparesis: efficacy, tolerability, and quality-of-life outcomes in a multicenter controlled trial. DOM-USA-5 Study Group. Clin Ther 1998; 20: 438-53.

16 Abell $\mathrm{T}$, McCallum R, Hocking M et al. Gastric electrical stimulation for medically refractory gastroparesis. Gastroenterology 2003; 125: 421-8.

17 Tack J, Talley NJ, Camilleri $\mathrm{M}$ et al. Functional gastroduodenal disorders. Gastroenterology 2006; 130: 1466-79.

18 Tougas G, Eaker EY, Abell TL et al. Assessment of gastric emptying using a low fat meal: establishment of international control values. Am I Gastroenterol 2000; 95: 1456-62.

19 Rentz AM, Kahrilas P, Stanghellini V et al. Development and psychometric evaluation of the patient assessment of upper gastrointestinal symptom severity index (PAGI-SYM) in patients with upper gastrointestinal disorders. Qual Life Res 2004; 13: 1737-49.

20 Revicki DA, Rentz AM, Dubois D et al. Development and validation of a patient-assessed gastroparesis symptom severity measure: the Gastroparesis Cardinal Symptom Index. Aliment Pharmacol Ther 2003; 18: 141-50.

21 De la Loge C, Trudeau E, Marquis P et al. Cross-cultural development and validation of a patient self-administered questionnaire to assess quality of life in upper gastrointestinal disorders: the PAGI-QOL. Qual Life Res 2004; 13: 1751-62.

22 Richter $\mathrm{P}$, Werner J, Heerlein A, Kraus A, Sauer H. On the validity of the beck depression inventory. A review. Psychopathology 1998; 31: 160-8.

23 Spielberger C, Gorsuch R, Lushene R. Manual for the State-Trait Anxiety Inventory. Palo Alto, CA: Consulting Psychologists Press, 1970.

24 Newcombe RG. Interval estimation for the difference between independent proportions: comparison of eleven methods. Stat Med 1998; 17: 873-90.

25 SAS software, version 9.3 of the SAS system for Windows, Cary, NC: SAS Institute, Inc, 2011.

26 Stata statistical software. release 11. College Station, TX: StataCorp, 2009.

27 Parkman HP, Yates K, Hasler WL et al. Clinical features of idiopathic gastroparesis vary with sex, body mass, symptom onset, delay in 
gastric emptying, and gastroparesis severity. Gastroenterology 2011; 140: 101-15.

28 Spiller R, Garsed K. Postinfectious irritable bowel syndrome. Gastroenterology 2009; 136: 1979-88.

29 Tack I, Demedts I, Dehondt G et al. Clinical and pathophysiological characteristics of acute-onset functional dyspepsia. Gastroenterology 2002; 122: 1738-47.

30 Hasler WL, Parkman HP, Wilson LA et al. Psychological dysfunction is associated with symptom severity but not disease etiology or degree of gastric retention in patients with gastroparesis. Am I Gastroenterol 2010; 105: 2357-67.

31 Van Oudenhove L, Vandenberghe J, Geeraerts B et al. Determinants of symptoms in functional dyspepsia: gastric sensorimotor function, psychosocial factors or somatisation? Gut 2008; 57: 1666-73.

32 Alok R, Das SK, Agarwal GG, Salwahan L, Srivastava R. Relationship of severity of depression, anxiety and stress with severity of fibromyalgia. Clin Exp Rheumatol 2011; 29(6 Suppl 69): S70-2.

33 Pasricha PJ, Colvin R, Yates K et al. Characteristics of patients with chronic unexplained nausea and vomiting and normal gastric emptying. Clin Gastroenterol Hepatol 2011; 9: 567-76.

34 Drossman DA, Toner BB, Whitehead WE et al. Cognitive-behavioral ther- apy versus education and desipramine versus placebo for moderate to severe functional bowel disorders. Gastroenterology 2003; 125: 19-31.

35 Tack J, Sarnelli G. Serotonergic modulation of visceral sensation: upper gastrointestinal tract. Gut 2002; 51 (Suppl 1): 77-80.

36 Revicki DA, Camilleri M, Kuo B, Szarka LA, McCormack J, Parkman HP. Evaluating symptom outcomes in gastroparesis clinical trials: validity and responsiveness of the Gastroparesis Cardinal Symptom Index-Daily Diary (GCSI-DD). Neurogastroenterol Motil 2012; 24: 456-63.

37 Maranki JL, Lytes V, Meilahn JE et al. Predictive factors for clinical improvement with Enterra gastric electric stimulation treatment for refractory gastroparesis. Dig Dis Sci 2008; 53: 2072-8.

38 Harrell SP, Studts JL, Dryden GW, Eversmann L, Cai L, Wo JM. A novel classification scheme for gastroparesis based on predominant-symptom presentation. I Clin Gastroenterol 2008; 42: 455-9.

39 MacGregor IL, Gueller R, Watts HD, Meyer JH. The effect of acute hyperglycemia on gastric emptying in man. Gastroenterology 1976; 70: 190-6.

40 Tack J, Caenepeel P, Fischler B, Piessevaux H, Janssens J. Symptoms associated with hypersensitivity to gastric distention in functional dyspepsia. Gastroenterology 2001; 121: 526-35.
41 Kumar A, Attaluri A, Hashmi S, Schulze KS, Rao SS. Visceral hypersensitivity and impaired accommodation in refractory diabetic gastroparesis. Neurogastroenterol Motil 2008; 20: 635-42.

42 Kuo B, Maneerattanaporn M, Lee AA et al. Generalized transit delay on wireless motility capsule testing in patients with clinical suspicion of gastroparesis, small intestinal dysmotility, or slow transit constipation. Dig Dis Sci 2011; 56: 2928-38.

43 Rao SS, Mysore K, Attaluri A, Valestin J. Diagnostic utility of wireless motility capsule in gastrointestinal motility. I Clin Gastroenterol 2011. 45: 684-90.

44 Abell TL, Familoni B, Voeller G et al. Electrophysiologic, morphologic, and serologic features of chronic nausea and vomiting: lessons learned from 121 consecutive patients. Surgery 2009; 145: 476-85.

45 Bromer MQ, Friedenberg F, Miller LS, Fisher RS, Swartz K, Parkman HP. Endoscopic pyloric injection of botulinum toxin A for the treatment of refractory gastroparesis. Gastrointest Endosc 2005; 61: 833-9.

46 Forstner-Barthell AW, Murr MM, Nitecki $S$ et al. Near-total completion gastrectomy for severe postvagotomy gastric stasis: analysis of early and long-term results in 62 patients. J Gastrointest Surg 1999; 3: 15-21.

\section{APPENDIX}

\section{Clinical centers}

Stanford University, Stanford, CA: Pankaj Jay Pasricha, MD (Principal Investigator); Marina Basina, MD; Jen Block, RN CDE; Bruce Buckingham, MD; Paula Clinton, RD CDE; Anna Karstens; Linda Nguyen, MD; Nighat Ullah, MD.

California Pacific Medical Center, San Francisco, CA: William Snape, MD (Principal Investigator); Nata DeVole, RN; Karen Earle, MD; Mary Greene, RN MS; Kjersti Kirkeby, MD; Corrie Nunez Lui, PA-C; Shelly Gray, RN MSN ANP-C; Katerina Shetler, MD.

Temple University, Philadelphia, PA: Henry P. Parkman, MD (Principal Investigator); Carol Homko, PhD RN; Steven Kantor; Vanessa Lytes, MSN CRNP; Amiya Palit, MD; Murali Pathikonda, MD; Priyanka Sachdeva, MD; Elias Siraj, MD; Kellie Simmons, NP.

Texas Tech University Health Sciences Center: Richard W. McCallum, MD (Principal Investigator); Tamis Bright, MD; Sean Connery; Iliana Faries; Reza Hejazi, MD; Joel Saldana, MD; Irene Sarosiek, MD; Denise Vasquez, RN; Natalia Vega, RN.

University of Michigan Health System, Ann Arbor, MI: William Hasler, MD (Principal Investigator); Michelle Atkinson, CSC; William Herman, MD; Amy E. Rothberg, MD; Sacha Uelman, RD CDE. 
University of Mississippi Medical Center, Jackson, MS: Thomas Abell, MD (Principal Investigator); Om Amin, MD; Niyutchai Chaithongdi, MD; JoAnne Fordham; Olivia Henry, MS RD; Archana Kedar, MD; Valerie McNair, LPN II; Natalie Moody; Shreya Patel, MD; Michelle Taylor.

Wake Forest University, Winston-Salem, NC: Kenneth Koch, MD (Principal Investigator); Lynn Baxter; Jorge Calles, MD; Samantha Culler; Judy Hooker; Lata Menon, MS RN CDE; Paula Stuart, PA.

\section{Resource Centers}

Mayo Clinic College of Medicine (Pathology Analyses Center), Rochester, MN: Gianrico Farrugia, MD (Principal Investigator); Cheryl Bernard.

National Institute of Diabetes, Digestive and Kidney Diseases, Bethesda, MD: Frank Hamilton, MD, MPH (Project Scientist); Steven James, MD; Patricia Robuck, PhD, MPH; Rebecca Torrance, RN MSN; Rebekah Van Raaphorst, $\mathrm{MPH}$.

Johns Hopkins University, Bloomberg School of Public Health (Data Coordinating Center), Baltimore, MD: James Tonascia, PhD (Principal Investigator); Patricia Belt; Michele Donithan, MHS; Mika Green, MA; Milana Isaacson; Wana Kim; Linda Lee, MD; Patrick May, MS; Laura Miriel; Alice Sternberg, ScM; Aynur Ünalp-Arida, MD, PhD; Mark Van Natta, MHS; Ivana Vaughn, MPH; Jessika Weldon, MPA; Laura Wilson, ScM; Katherine Yates, ScM. 